\title{
Purificación de dióxido de carbono emitido en plantas de tratamiento de gas
}

\author{
Purification of the carbon dioxide \\ emitted by gas treatment plants
}

Fecha de Recepción: 22 - oct. - 2017.

Fecha de Aceptación: 16 - may. - 2018.

\section{Resumen}

En las plantas de extracción de gas natural, el dióxido de carbono $\left(\mathrm{CO}_{2}\right)$ que viaja en la corriente de gas extraída, se separa y se libera a la atmósfera, acompañado de sulfuro de hidrógeno $\left(\mathrm{H}_{2} \mathrm{~S}\right)$, agua e hidrocarburos. El objetivo de esta investigación fue diseñar un proceso de purificación de la corriente de $\mathrm{CO}_{2}$ residual, de tal manera que se evite su emisión a la atmósfera y se pueda utilizar en la industria alimenticia. En la industria alimenticia, el $\mathrm{CO}_{2}$ se puede usar en bebidas carbonatadas y como fluido supercrítico, requiriendo una pureza superior a 99,95\% mol. Para ello, se seleccionó como caso de estudio un flujo real de 3454 ton/año de $\mathrm{CO}_{2}$ emitido por una planta de tratamiento de gas localizada en Neiva, Colombia y se simularon varios procesos de purificación usando el software ProMax. La purificación y recuperación de $\mathrm{CO}_{2}$ se evaluó mediante (1) absorción química con metildietanolamina (MDEA), (2) deshidratación con trietilenglicol (TEG) combinada con tamiz molécular, y (3) remoción de compuestos livianos (metano-etano) por el proceso criogénico de Ryan Holmes. La combinación de los tres procesos permitió una recuperación de 99,95\% de $\mathrm{CO}_{2}$ con una pureza del 99,99\% mol, requiriendo una inversión total de USD 412.323 o $53 \mathrm{USD} / \mathrm{tCO}_{2}$. Este $\mathrm{CO}_{2}$ podría ser comercializado a valores superiores a 5000 $\mathrm{USD} / \mathrm{tCO}_{2}$ en la industria alimenticia. Además, la combinación de los procesos (1) y (2) arrojó costos de 37 $\mathrm{USD} / \mathrm{tCO}_{2}$, produciendo una pureza de $\mathrm{CO}_{2}$ de $99,85 \% \mathrm{~mol}$, apto para otras aplicaciones de menor pureza en la industria alimenticia o en el recobro mejorado del petróleo.

Palabras clave: Calentamiento global; CAPEX; Costos de inversión; Captura y uso de carbono; Simulación de procesos.

a Magíster en Diseño y Gestión de Procesos. Grupo de Energía, Materiales y Ambiente, Facultad de Ingeniería. Universidad de La Sabana. Campus Universitario Puente del Común. Chía, Colombia / Ingeniera de Procesos. Stoc (MASA), Bogotá, Colombia.

b Doctora en Ingeniería. Grupo de Energía, Materiales y Ambiente, Facultad de Ingeniería. Universidad de La Sabana. Campus Universitario Puente del Común. Chía, Colombia. Correo electrónico: martha.cobo@ unisabana.edu.co. 


\begin{abstract}
In natural gas plants, the carbon dioxide $\left(\mathrm{CO}_{2}\right)$ traveling in the extracted stream is separated and released to the atmosphere. This stream also contains hydrogen sulfide $\left(\mathrm{H}_{2} \mathrm{~S}\right)$, water and hydrocarbons. The purpose of this investigation was to design a process to purify this $\mathrm{CO}_{2}$ stream, to its further use in the food industry. There, the $\mathrm{CO}_{2}$ can be employed in carbonated drinks and as supercritical fluid; requiring a purity higher than $99.95 \mathrm{~mol} \%$. Therefore, a real flow of 3454 ton/year $\mathrm{CO}_{2}$ was selected as case of study, which is emitted in a gas treatment plant located in Neiva, Colombia. This stream was simulated in ProMax and subjected to chemical absorption with methyl diethanolamine (MDEA), to a dehydration with diethylene glycol combined with molécular sieve, to a Ryan-Holmes cryogenic process for removing light components (methane and ethane) and their combinations. The combination of the three processes allowed us for a $99.95 \% \mathrm{CO}_{2}$ recovery and a $99.99 \mathrm{~mol}^{\%} \mathrm{CO}_{2}$ purity, requiring capital expenses (CAPEX) of USD 412.323 or $53 \mathrm{USD} / \mathrm{tCO}_{2}$. This $\mathrm{CO}_{2}$ could be commercialized at sell prices as high as $5000 \mathrm{USD} /$ $\mathrm{tCO}_{2}$ to the food industry. In addition, the combination of the two first processes delivered a CAPEX of 37 $\mathrm{USD} / \mathrm{tCO}_{2}$ with a $99.85 \mathrm{~mol} \% \mathrm{CO}_{2}$ purity, suitable for other applications requiring less purity in the food industry or improved oil recovery.
\end{abstract}

Key words: Global warming; CAPEX; Capital expenses; Carbon capture and utilization (CCU); Process simulation.

\section{INTRODUCCIÓN}

En Colombia (Suramérica), el número de plantas de tratamiento de gas (TG) ha aumentado rápidamente en los últimos años. De acuerdo con el Reglamento Único de Transporte de Colombia (Resolución 071 [1]), el gas natural extraído se debe tratar para su posterior transporte hasta obtener un contenido de dióxido de carbono $\left(\mathrm{CO}_{2}\right)$ y sulfuro de hidrógeno $\left(\mathrm{H}_{2} \mathrm{~S}\right)$ inferiores a $2 \% \mathrm{~mol}$ y 4 ppm, respectivamente. El proceso de absorción con aminas es uno de los más utilizados en las plantas de TG para la eliminación de estos compuestos ácidos, donde usualmente se emplea una torre de absorción [2]. La metildietanolamina (MDEA) es una amina terciaria muy utilizada en este proceso. La etapa de regeneración tiene 4 etapas principales: expansión, filtración, calentamiento y regeneración. La desorción del $\mathrm{CO}_{2}$ se produce en la última etapa del proceso en un separador, donde el $\mathrm{CO}_{2}$ se libera a la atmósfera [3]. De esta manera, estas plantas liberan altas cantidades de $\mathrm{CO}_{2}$ que podrían ser capturadas [4] y utilizadas en varias aplicaciones, con el fin de proporcionar una alternativa para reducir las emisiones de gases de efecto invernadero (GEI) [5].

Sin embargo, las condiciones de captura dependen de las concentraciones de $\mathrm{CO}_{2}$ y la presión de la corriente, y se requerirán tecnologías diferentes para el funcionamiento óptimo en términos de energía y costo. Por ejemplo, para una corriente de gas que contiene elevadas cantidades de agua saturada, se requiere la remoción de esta agua [6]. Para ello, la deshidratación con trietilenglicol (TEG) es el proceso más utilizado en la industria del petróleo y el gas (O\&G), que se combina con un proceso de adsorción para la remoción de agua remanente [7]. Por otra parte, esta corriente viaja con $\mathrm{H}_{2} \mathrm{~S}$ que puede eliminarse mediante un proceso de absorción con MDEA [2]. Este solvente es selectivo para eliminar $\mathrm{H}_{2} \mathrm{~S}$ y los requerimientos energéticos para su regeneración son bajos [3].

Por otro lado, los procesos criogénicos pueden producir corrientes de $\mathrm{CO}_{2}$ altamente puras, pero deben considerar el uso de refrigeración y alto consumo de energía [8]. Los tamices moléculares también se pueden usar como desecantes para deshidratar las corrientes de $\mathrm{CO}_{2}$ [9]. Los adsorbentes como cloruro de calcio $\left(\mathrm{CaCl}_{2}\right)$, silicatos de aluminio y tamices moléculares se pueden usar como desecantes consumibles para deshidratar $\mathrm{CO}_{2}$.

El propósito de este estudio fue establecer un diseño de proceso que permita la purificación del $\mathrm{CO}_{2}$ emitido a la atmósfera desde el sistema de endulzamiento de gas natural en plantas de TG para su posterior comercialización. Esto con el fin de ofrecer una alternativa para reducir las emisiones de GEI y generar un mecanismo de desarrollo limpio en el proceso de una planta de TG. En la industria alimenticia, el $\mathrm{CO}_{2}$ se puede 
utilizar en bebidas carbonatadas y como fluido supercrítico, requiriendo una pureza de entre 99,5 y $99,9 \%$ mol. Por esta razón, se simularon diferentes configuraciones y combinaciones de etapas de proceso, utilizando ProMax como simulador de procesos, para determinar la tecnología más adecuada considerando la recuperación y pureza de $\mathrm{CO}_{2}$, fácil operación y costo.

\section{MATERIALES Y MÉTODOS}

\subsection{Planta de tratamiento de gas}

En este estudio se analizó una planta de TG con una capacidad de $11780 \mathrm{sm}^{3} / \mathrm{h}$ localizada en la ciudad de Neiva, Colombia (latitud: 2,934484 y longitud: 75,2804). Esta planta libera $1177 \mathrm{sm}^{3} / \mathrm{h}$ de $\mathrm{CO}_{2}$ a la atmósfera, y tiene un proceso de ocho etapas descrito en la Figura 1. La primera etapa es un separador trifásico primario (1), donde se obtienen tres corrientes: agua, hidrocarburos pesados y gas agrio. La compresión mediante el uso de compresores reciprocantes es la segunda etapa (2). El endulzamiento de gas es la tercera etapa
(3) y se compone de bombas centrífugas, tanque flash, intercambiador de placas, válvulas de control de nivel y temperatura, torres de contacto y regeneración. El sistema de glicol es la cuarta etapa (4), también basado en la absorción química, que elimina el agua del gas para prevenir la formación de hidratos. La siguiente etapa (5) es el sistema de ajuste de punto de rocío, donde se efectúa la licuefacción del gas utilizando los principios de Joule Thompson y refrigeración mecánica, para disminuir el contenido de compuestos pesados $(\mathrm{C} 3+)$. El sistema de fraccionamiento es la etapa posterior (7), donde una torre deetanizadora separa el remanente de $\mathrm{CH}_{4}$ y etano de la corriente líquida proveniente del sistema de ajuste de punto de rocío; para cumplir con el parámetro de concentración de etano $(<6 \% \mathrm{~mol})$. El gas que se obtiene en el sistema de punto de rocío y en el tope de la torre deetanizadora se envía como gas de venta; mientras que los líquidos pasan a una torre de estabilización de condensados (8), y en esta se generan gas licuado de petróleo (LPG) y líquidos de gas natural (LGN).

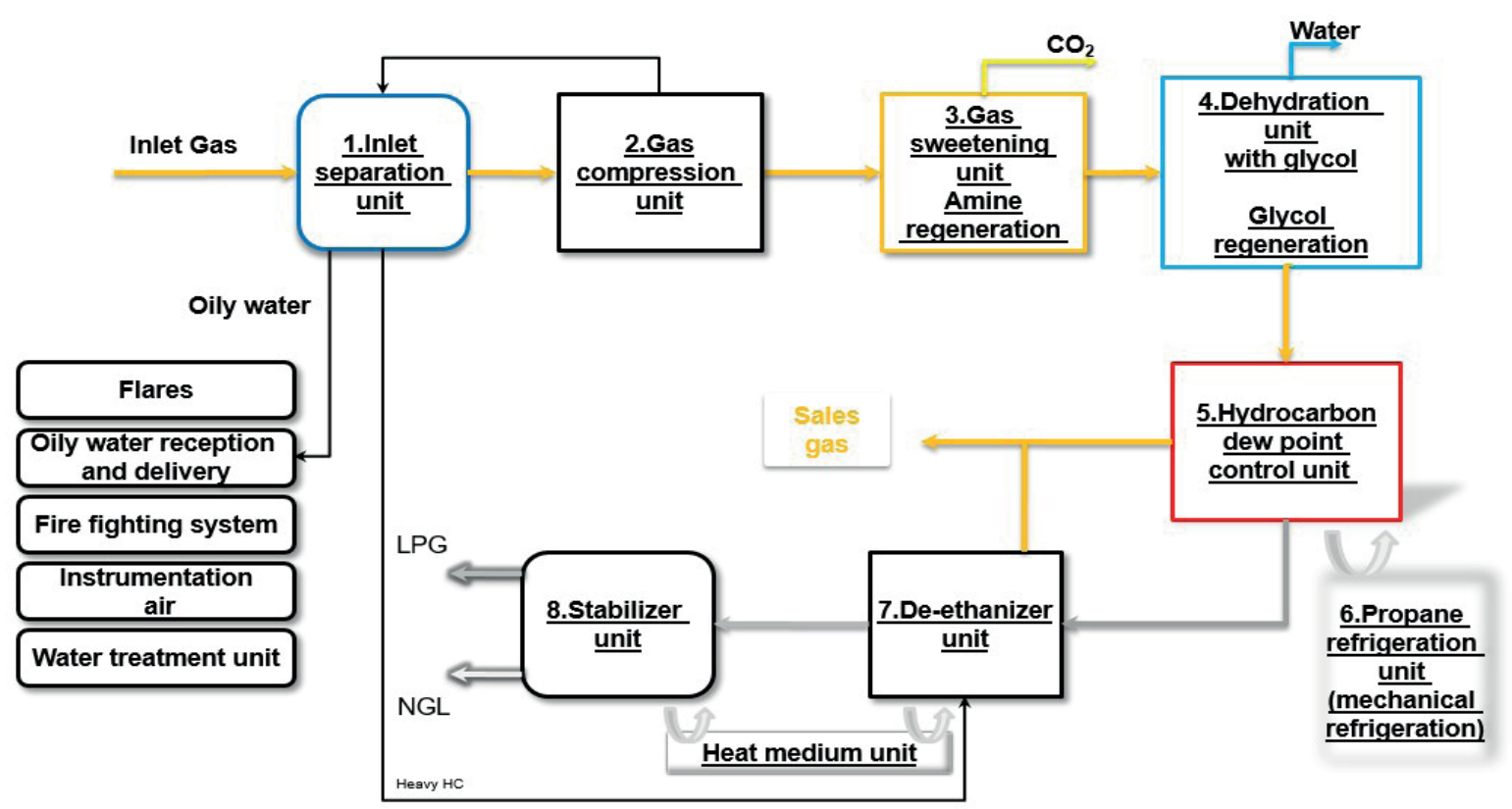

Figura 1. Diagrama de flujo de la planta de TG utilizada como caso de estudio. 


\subsection{Caracterización de la corriente de $\mathrm{CO}_{2}$ a purificar}

Se tomaron muestras de la corriente de gas ácido de la torre de regeneración del sistema de endulzamiento de la planta de TG (corriente de $\mathrm{CO}_{2}$ en la Figura 1). Las condiciones de operación de la torre de regeneración de amina en el momento del muestreo fueron, en promedio, $115^{\circ} \mathrm{C}$ en los fondos, $97{ }^{\circ} \mathrm{C}$ en la parte superior y $62 \mathrm{kPa}$ de presión. Las muestras de gas ácido se analizaron en un cromatógrafo de gases 7890 A GC 2010 (Agilent, USA) equipado con una columna capilar de tipo fibra de vidrio $(50 \mathrm{~m}$ de longitud, 0,53 $\mathrm{mm}$ de diámetro interno, $0,5 \mu \mathrm{m}$ de espesor de película), conectada a un detector de ionización de llama (FID); y dos columnas tipo acero inoxidable $(50 \mathrm{~m}, 0,5 \mu \mathrm{m}$, Agilent, USA) rellenas con tierra de diatomeas, conectadas a un detector de conductividad térmica (TCD). Además, se midió la humedad y la concentración de $\mathrm{H}_{2} \mathrm{~S}$ en la corriente de gas ácido con un sistema Dragger (Colombia) usando el principio de medición colorimétrica.

\subsection{Simulación de los procesos de purificación de $\mathrm{CO}_{2}$}

La purificación de $\mathrm{CO}_{2}$ se simuló utilizando tres procesos: (1) eliminación de $\mathrm{H}_{2} \mathrm{~S}$ por absorción con aminas, (2) deshidratación con TEG y (3) sistema criogénico Ryan Holmes para remoción de compuestos livianos (metano-etano). Cada tecnología se explica a continuación.

\subsubsection{Proceso 1: Eliminación de $\mathrm{H}_{2} \mathrm{~S}$ por absorción con aminas}

La amina utilizada para este proceso fue MDEA, que se tomó del sistema de endulzamiento de la planta de TG (proceso 3 en la Figura 1), que normalmente funciona con un flujo de $0,23 \mathrm{~m}^{3} / \mathrm{min}$, pero puede trabajar con $0,19 \mathrm{~m}^{3 /}$ min sin aumentar la carga ácida de la solución. Por tanto, se tomaron $0,037 \mathrm{~m}^{3} / \mathrm{min}$ de MDEA del sistema principal de amina y se recircularon al proceso de purificación de $\mathrm{CO}_{2}$, para eliminar $\mathrm{H}_{2} \mathrm{~S}$ de la corriente de gas ácido (corriente de $\mathrm{CO}_{2}$ en la Figura 1). Ambos procesos, primario y secundario, se simularon usando ProMax (Versión 3.2, Bryan Research Engineering). El paquete termodinámico para el endulzamiento de amina fue Soave-Redlich-Kwong (SRK), el cual predice las propiedades de la fase líquida por el modelo de líquido de reacción electrolítica (ELR) y las propiedades de la fase de vapor utilizando las ecuaciones de estado de Soave-Redlich-Kong (SRK) [10]. La Figura 2 muestra el sistema simulado. La corriente de $\mathrm{CO}_{2}$ entra en una torre de contacto (S1) de 6 etapas teóricas; estas son las mismas etapas teóricas de la torre real del proceso de endulzamiento de la planta de TG. Se tomaron las mismas etapas teóricas porque un número menor de etapas disminuye el tiempo de residencia y, por tanto, la absorción de $\mathrm{H}_{2} \mathrm{~S}$. La relación de etapas reales a etapas ideales fue de 3 , según el modelo cinético TSWEET de ProMax, que busca un tiempo de residencia apropiado del líquido en la columna.

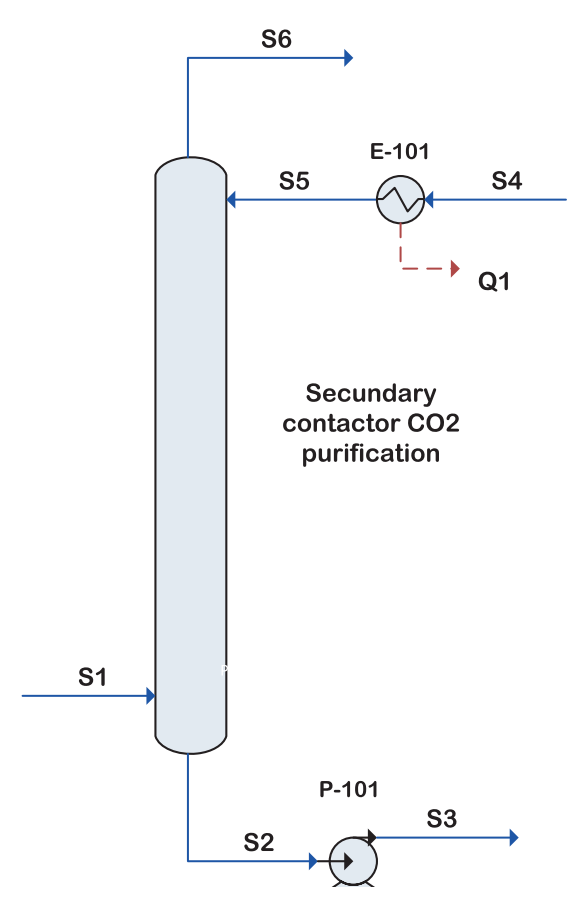

Figura 2. Diagrama de flujo del proceso diseñado en ProMax para la absorción de $\mathrm{H}_{2} \mathrm{~S}$. Las líneas azules continuas representan corrientes de materia y las líneas rojas punteadas representan corrientes de energía. Notación del equipo: P: Bomba, E: Intercambiador de calor.

De acuerdo con la Figura 2, la solución de amina de la planta de proceso de amina primaria se enfría y entra en la parte superior de la torre (S5). La amina en el fondo de la torre se envía a la etapa de amina primaria a regenerar (S3). Este proceso es posible debido a que la solución de amina usada se toma del proceso principal de endulzamiento de la planta de estudio y esto disminuye el costo de implementar un sistema de 
regeneración para esta tecnología. Este proceso de configuración se estableció así para conseguir la eliminación del $\mathrm{H}_{2} \mathrm{~S}$ de la corriente de $\mathrm{CO}_{2}$.

\subsubsection{Proceso 2: Deshidratación con TEG}

La Figura 3 muestra el proceso requerido para reducir el agua en la corriente de $\mathrm{CO}_{2}$. El paquete de propiedades termodinámicas utilizado fue la ecuación de estado de Redlich-Kwong modificada por Soave, que puede describir las fases líquidas y de vapor y es adecuada para sustancias polares [9]. ProMax recomienda para el proceso de deshidratación, que la pureza típica del TEG después de la regeneración sea de $98,7 \%$ p/p y la temperatura del regenerador de glicol está limitada por la degradación térmica del TEG $\left(204{ }^{\circ} \mathrm{C}\right)$. Martínez [9] mostró que las bandejas de burbujas en un sistema TEG/gas tienen un $25 \%$ de eficiencia total. En consecuencia, las etapas teóricas se dividieron por la eficiencia global para el cálculo de las etapas reales, obteniendo 28 etapas reales.

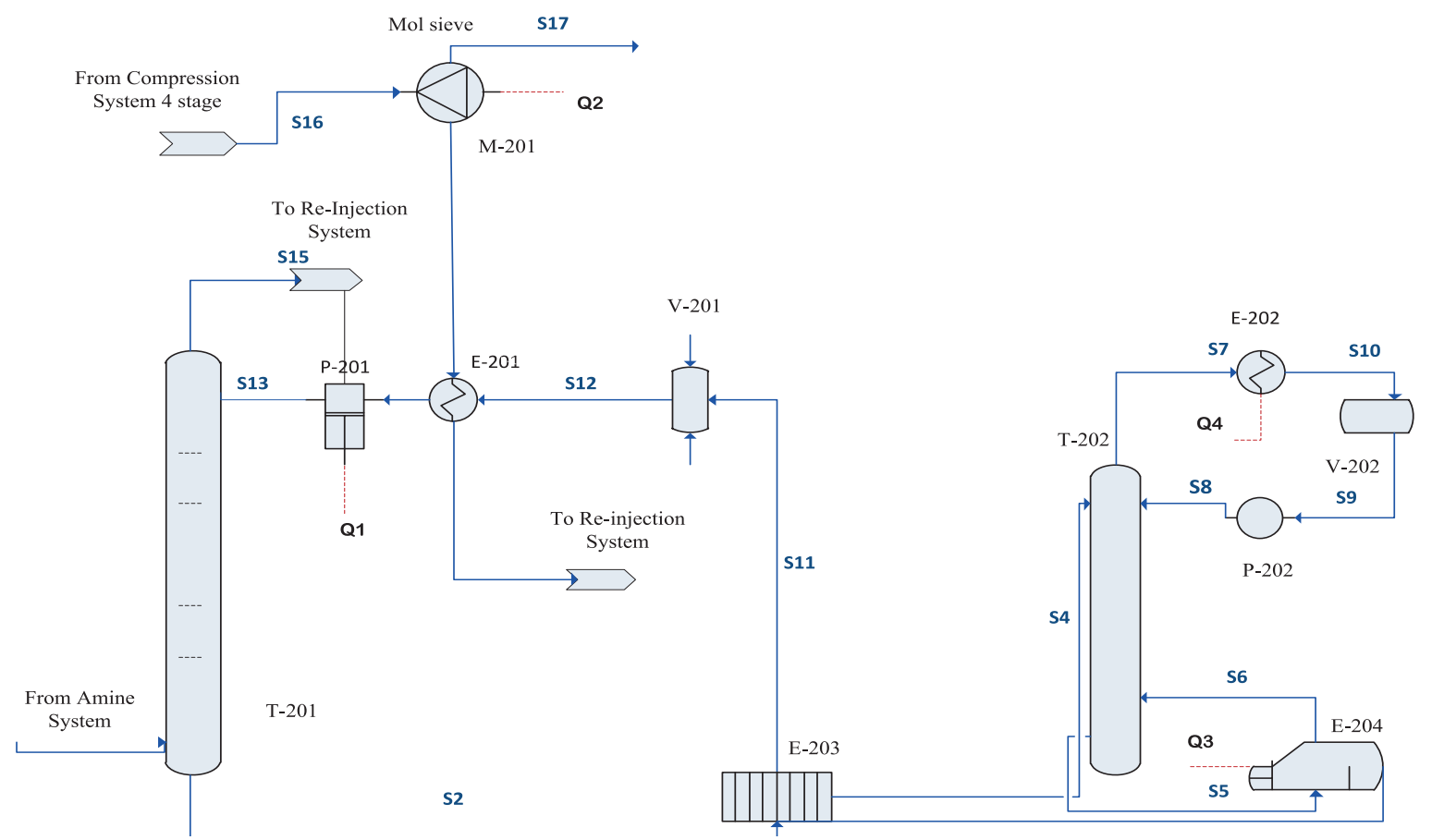

Figura 3. Diagrama de flujo del proceso diseñado en ProMax para deshidratación. Las líneas azules continuas representan corrientes de materia y las líneas rojas punteadas representan corrientes de energía. Notación del equipo: P: Bomba, E: Intercambiador de calor, V: Vasija, M: Mol Sieve, T: Torre.

En la Figura 3, el gas entra por el fondo de la torre (desde el sistema de amina de la Figura 2) y el TEG entra en la parte superior (S13). El glicol rico (S2) sale del fondo de la torre y pasa a través de un intercambiador de placas (glicol rico/pobre), donde intercambia calor con el glicol pobre para aumentar la temperatura. La solución de glicol regenerado (S12) pasa a través del intercambiador de calor de placas y luego se enfría con el gas de proceso y vuelve a la etapa de absorción (S13). Por su lado, el gas parcialmente saturado sale de la parte superior de la torre
(S15). El gas a alta presión vuelve a circular a la etapa de deshidratación (S16), y a continuación, pasa a través del tamiz molécular. El diseño de un sistema de deshidratación con desecantes implica: la cantidad requerida de desecante, el diámetro y la longitud del lecho y el suministro de calor necesario para la regeneración del lecho (S17). La vasija V-201 cumple la función de sistema Make-up, que se usa en el proceso en planta para realizar las reposiciones de glicol, cuando se generan pérdidas por arrastre o para aumentar la concentración de glicol en la mezcla. 


\subsubsection{Proceso 3: Sistema criogénico Ryan Holmes}

La Figura 4 muestra el diagrama de flujo del proceso Ryan Holmes, en el que el gas seco entra a alta presión y libre de agua. La torre de fraccionamiento tiene 6 etapas teóricas y 24 etapas reales. El paquete termodinámico utilizado fue Peng-Robinson. Esta ecuación de estado da resultados similares a los de Soave, sin embargo, es más precisa para predecir las densidades de los compuestos en fase líquida, especialmente no polares [11]. El gas sufre una caída de presión y pasa a través del intercambiador de calor, donde la temperatura cae a $-40{ }^{\circ} \mathrm{C}(\mathrm{Sr} 2)$. Después de esto, pasa a una torre de fraccionamiento, donde los compuestos orgánicos volátiles como metano y etano se separan $(\mathrm{Sr} 5)$ y el $\mathrm{CO}_{2}$ sale en la parte inferior como líquido (Sr10).

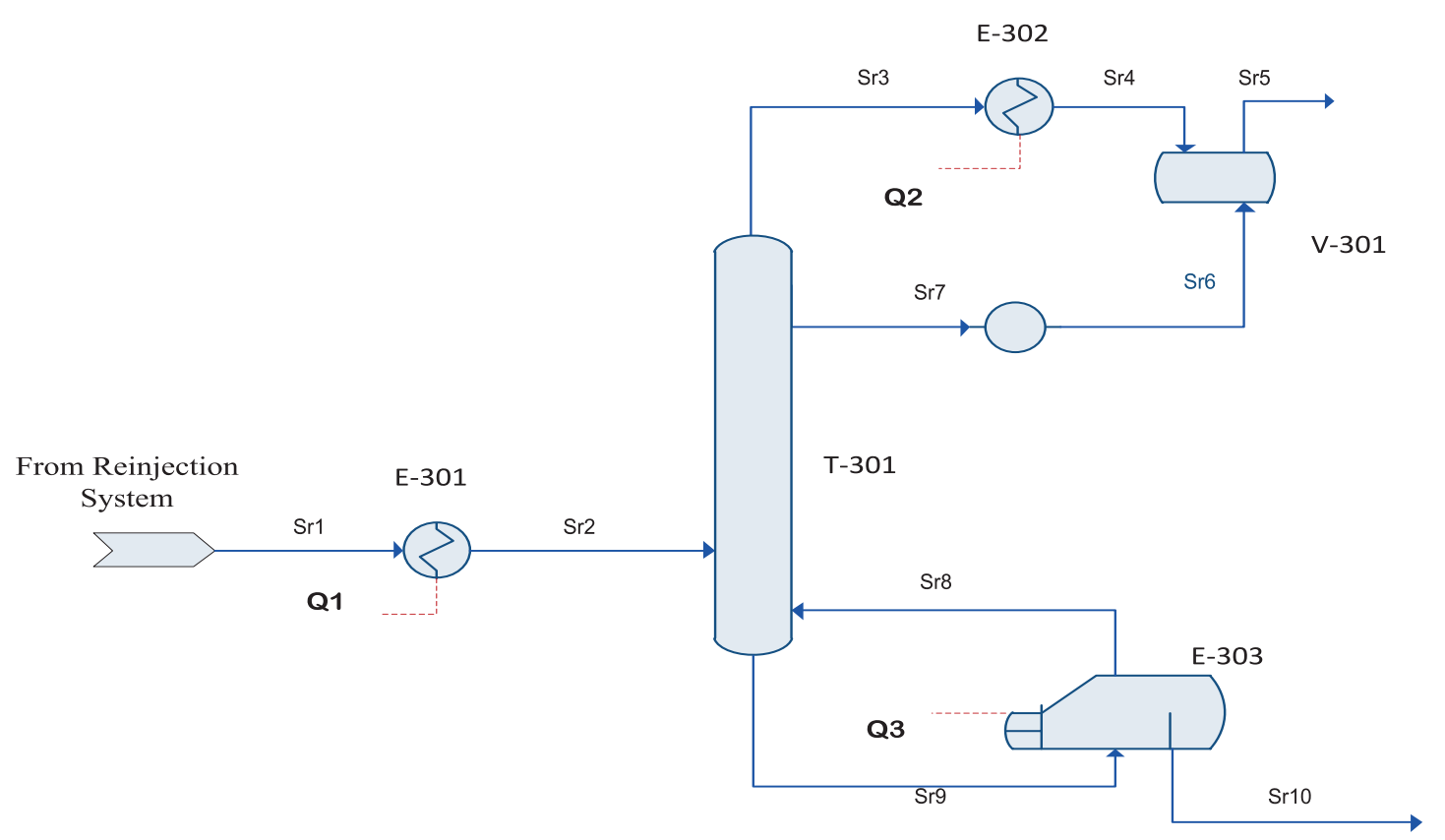

Figura 4. Diagrama de flujo del proceso Ryan Holmes diseñado en ProMax. Las líneas azules continuas representan corrientes de materia y las líneas rojas punteadas representan corrientes de energía. Notación del equipo: E: Intercambiador de calor, V: Vasija, T: Torre.

\subsection{Recuperación y pureza de $\mathrm{CO}_{2}$}

La eficiencia de cada sistema se determinó mediante un balance de masa en cada tecnología, dadas las entradas y salidas. Se evaluó la recuperación de $\mathrm{CO}_{2}$, de acuerdo con la ecuación 1, donde los flujos están en $\mathrm{kmol} / \mathrm{h}$, y la pureza de dicha corriente se contrastó con el valor requerido en la industria alimenticia, de mínimo 99,95\% mol.

$$
\text { Recuperación de } \mathrm{CO}_{2}=\frac{\text { Salida de } \mathrm{CO}_{2}\left(\frac{\mathrm{kmol}}{\mathrm{h}}\right)}{\text { Entrada de } \mathrm{CO}_{2}\left(\frac{\mathrm{kmol}}{\mathrm{h}}\right)} \quad \text { Ec. (1) }
$$

\subsection{Costos de operación}

La evaluación de la factibilidad económica de cada planta simulada se realizó mediante una evaluación económica clase $\mathrm{V}$, de acuerdo a la American Association of Cost Engineers [12]. Estas evaluaciones generalmente se realizan para evaluar proyectos en etapa de concepto y producir estimaciones de costos totales con una precisión de predicción de entre 30 y $50 \%$. Todas las estimaciones de costos se relacionan como costos de capital (Capex). Los costos de cada proceso se estimaron teniendo en cuenta todos los equipos y los servicios auxiliares. Por ejemplo, para estimar el costo de las bombas se tuvo en cuenta el tipo rotativo y alternativo. Para el costo de las torres de absorción, el diámetro y el número de placas teóricas. Y para el costo de los intercambiadores 
de calor, el área de transferencia de calor y la energía interna [13]. Los cálculos de CAPEX se realizaron siguiendo la metodología descrita por Seider y col. [14], donde para cada tecnología se estimó el costo total y los servicios auxiliares asociados. Los cálculos de la torre de absorción en el proceso de deshidratación se realizaron mediante la metodología reportada por [9]. Los costos estimados se actualizaron a 2016 usando una tasa promedio de incremento de $1,2 \%$ anual del producto interno bruto (PIB) reportado por el banco mundial [15].

\section{RESULTADOS Y ANÁLISIS}

\subsection{Caracterización experimental de la corriente de $\mathrm{CO}_{2}$}

Tabla 1. Caracterización de la corriente de $\mathrm{CO}_{2}$ emitida en la planta de endulzamiento de gas natural

\begin{tabular}{cccc}
\hline \multirow{2}{*}{ Componentes } & \multicolumn{3}{c}{ Concentración $(\% \mathrm{~mol})$} \\
\cline { 2 - 4 } & 1 & 2 & 3 \\
\hline $\mathrm{CO}_{2}$ & 91,67 & 70,19 & 91,82 \\
Metano & 0,00 & 0,25 & 0,00 \\
Ethano & 1,17 & 0,00 & 1,17 \\
Propano & 0,09 & 0,00 & 0,09 \\
i-Butano & 0,00 & 0,00 & 0,00 \\
n-Butano & 0,00 & 0,00 & 0,00 \\
i-Pentano & 0,00 & 0,00 & 0,00 \\
n-Pentano & 0,00 & 0,00 & 0,00 \\
$\mathrm{O}_{2}$ & 0,59 & 6,23 & 0,59 \\
$\mathrm{~N}_{2}$ & 0,06 & 17,33 & 0,06 \\
n-Hexano & 0,00 & 0,00 & 0,00 \\
$\mathrm{H}_{2} \mathrm{O}$ & 6,42 & 6,00 & 6,26 \\
$\mathrm{H}_{2} \mathrm{~S}$ & $276 \mathrm{ppm}$ & $200 \mathrm{ppm}$ & $250 \mathrm{ppm}$ \\
\hline
\end{tabular}

Flujo total $=1177 \mathrm{~m}^{3} / \mathrm{h}, \mathrm{T}=44^{\circ} \mathrm{C}$ y P $=62 \mathrm{kPa}$

Debido a las variaciones en la concentración del gas de entrada a la planta de (TG), se realizaron varias mediciones del subproducto de $\mathrm{CO}_{2}$ del proceso de endulzamiento en un mes para encontrar las mejores condiciones de funcionamiento, flujo no perturbado, cambios de presión y concentración en el flujo de entrada que entra a la planta de gas. Los resultados de tres muestras típicas se muestran en la Tabla 1. La variación en la concentración de las muestras depende de las condiciones de funcionamiento del sistema de regeneración en la planta de TG, porque cuando la temperatura aumenta en la parte superior de la torre, se genera un arrastre de amina en la corriente de $\mathrm{CO}_{2}$ emitida, debido a la desestabilización de la planta. De este modo, se seleccionó la muestra 1, que contenía $92 \%$ mol de $\mathrm{CO}_{2}, 6,4 \%$ mol de agua y $276 \mathrm{ppm}$ de $\mathrm{H}_{2} \mathrm{~S}$ (esta composición se tomó como corriente de entrada para el proceso de purificación). Estas condiciones corresponden a los tiempos más estables de la planta sin que se hayan presentado perturbaciones en el proceso que pudieran alterar la medición.

\subsection{Simulación de los sistemas de recuperación y purificación de $\mathrm{CO}_{2}$}

\subsubsection{Proceso 1: Eliminación de $\mathrm{H}_{2} \mathrm{~S}$ por absorción con aminas}

Se empleó absorción con MDEA para eliminar el $\mathrm{H}_{2} \mathrm{~S}$ que viajaba con la corriente de $\mathrm{CO}_{2}$ a la salida del sistema de endulzamiento de gas, utilizando la composición de la muestra 1 de la Tabla 1 y el proceso descrito en la Figura 2. Se estudiaron dos variables de proceso, la temperatura del solvente y la presión del gas de entrada a la torre de contacto. Utilizando ProMax, se varió la temperatura del solvente entre 21 y $60{ }^{\circ} \mathrm{C}$ y la presión de gas entre 138 y $379 \mathrm{kPa}$. Los resultados se muestran en la Figura 5. El punto más alto de recuperación y pureza de $\mathrm{CO}_{2}, \mathrm{y}$ la eliminación de $\mathrm{H}_{2} \mathrm{~S}$, se obtuvo a $21{ }^{\circ} \mathrm{C}$ y 138 $\mathrm{kPa}$, estas condiciones de operación favorecen la reacción exotérmica entre la amina y el $\mathrm{H}_{2} \mathrm{~S}$. La presión en el gas de entrada y la temperatura del solvente afectaron inversamente la absorción de $\mathrm{H}_{2} \mathrm{~S}$. La temperatura del solvente que entra a la torre de contacto debe ser $10{ }^{\circ} \mathrm{C}$ mayor que la temperatura del gas, para evitar la condensación de hidrocarburos [16]. Sin embargo, en este caso, y considerando que la corriente de gas tiene una alta concentración de compuestos ácidos, la temperatura del solvente podría reducirse a $23{ }^{\circ} \mathrm{C}$. Para este caso, se verificó la viscosidad del solvente para determinar a qué temperatura dejaría de fluir, lo que ocurrió a $15^{\circ} \mathrm{C}$. 
(a)

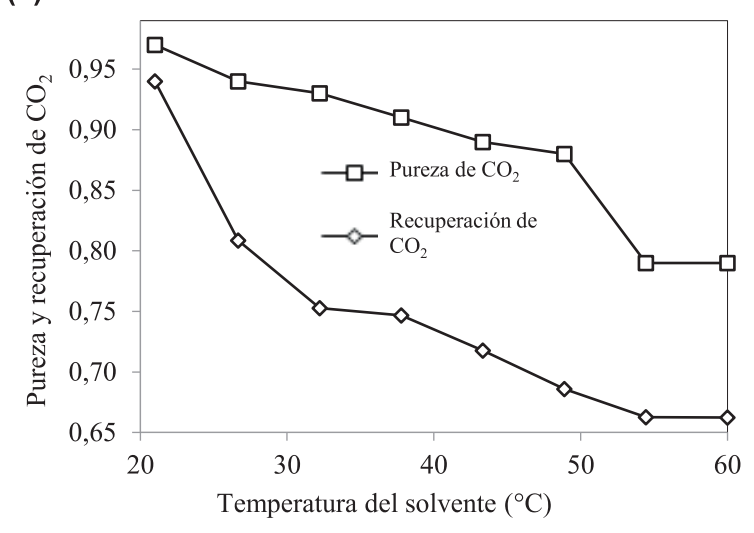

(b)

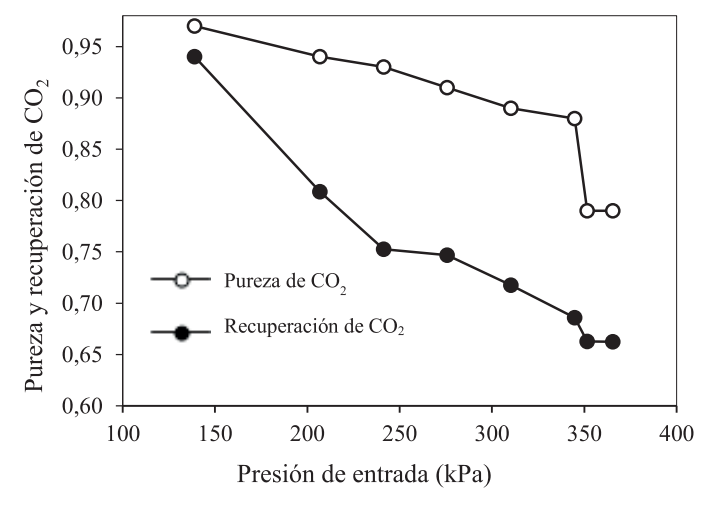

(c)

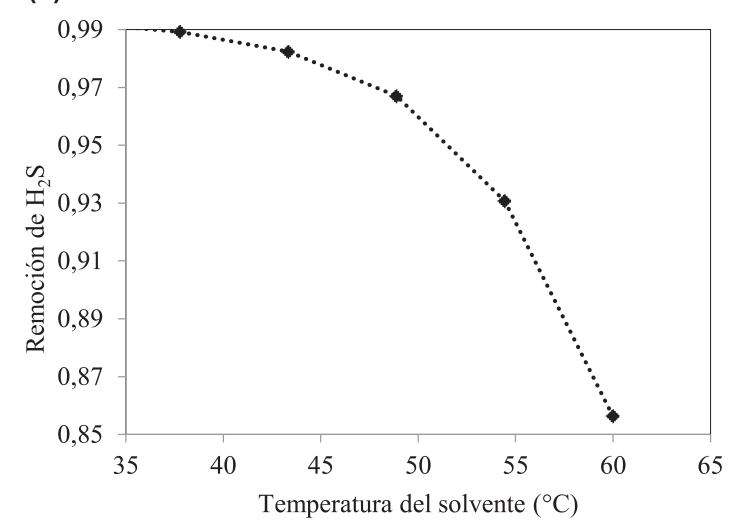

Figura 5. Efecto de (a) la temperatura del solvente, (b) de la presión de entrada de gas y (c) la temperatura del solvente sobre la recuperación y la pureza de $\mathrm{CO}_{2}$ durante la eliminación de $\mathrm{H}_{2} \mathrm{~S}$ por absorción con MDEA.

\subsubsection{Proceso 2: Sistema de deshidratación con TEG}

El contenido de agua en la corriente de $\mathrm{CO}_{2}$ mostrado en la Tabla 1, se eliminó por deshidratación con TEG, de acuerdo con el proceso de la Figura 3. Para ello, se varió la temperatura del rehervidor de la torre de destilación entre 149 y $204{ }^{\circ} \mathrm{C}$, hasta alcanzar la concentración de TEG más alta en la torre de regeneración. De esta manera, se obtuvo una mayor absorción de agua libre en el gas debido a que, a mayor concentración de glicol en la solución regenerada, mayor su capacidad de absorción. Adicionalmente, para eliminar la cantidad de agua total de la corriente de gas, se incluyó un tamiz molécular a la salida del tope de la torre contactora del sistema de deshidratación, donde el gas pasó a través del lecho adsorbente y retuvo selectivamente las moléculas de agua, hasta llevar la concentración de agua a $0 \%$ mol en la corriente S17 [6].

\subsubsection{Proceso 3: Sistema criogénico Ryan Holmes}

La tecnología Ryan Holmes de la Figura 4 se empleó para purificar el $\mathrm{CO}_{2}$ por procesos criogénicos. La Figura 6 muestra el efecto de variar la temperatura y la presión de la corriente de entrada al proceso sobre la recuperación y pureza de $\mathrm{CO}_{2}$. Para obtener la máxima recuperación de $\mathrm{CO}_{2}$ en la parte inferior de la torre, se requirió una presión de $2758 \mathrm{kPa}$ y una temperatura en el condensador de $-40{ }^{\circ} \mathrm{C}$. Para la torre de fraccionamiento, se establecieron 6 etapas teóricas, teniendo en cuenta las condiciones de diseño definidas en el simulador de procesos (ProMax) para procesos criogénicos. El aumento del número de las etapas de la torre no varió la recuperación de $\mathrm{CO}_{2}$ ni el perfil de la torre, ya que este proceso estaba en su máximo punto operativo. El método CDC (condensación y destilación criogénica) se ha empleado para la eliminación de $\mathrm{CO}_{2}$ en mezclas de $\mathrm{CH}_{4} / \mathrm{CO}_{2}$ y otros gases usados en la recuperación de hidrocarburos de fuentes naturales. Este proceso requiere un consumo elevado de energía cuando la concentración de $\mathrm{CO}_{2}$ en los gases de entrada es baja [8]. 
(a)

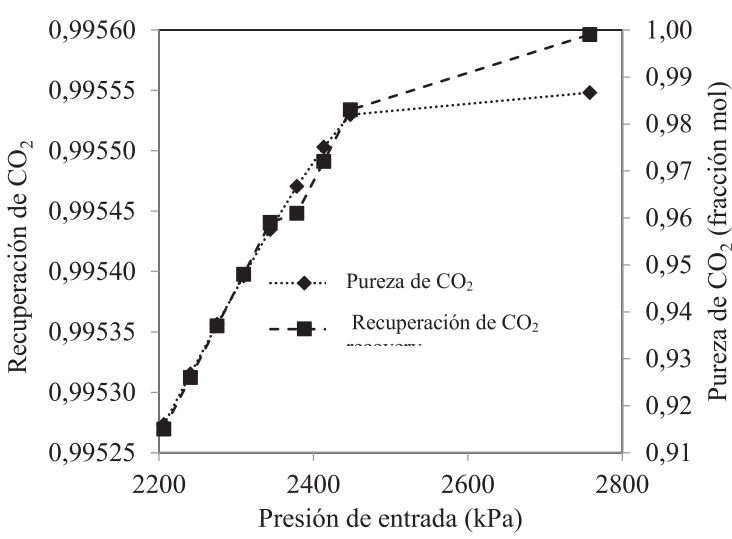

(b)

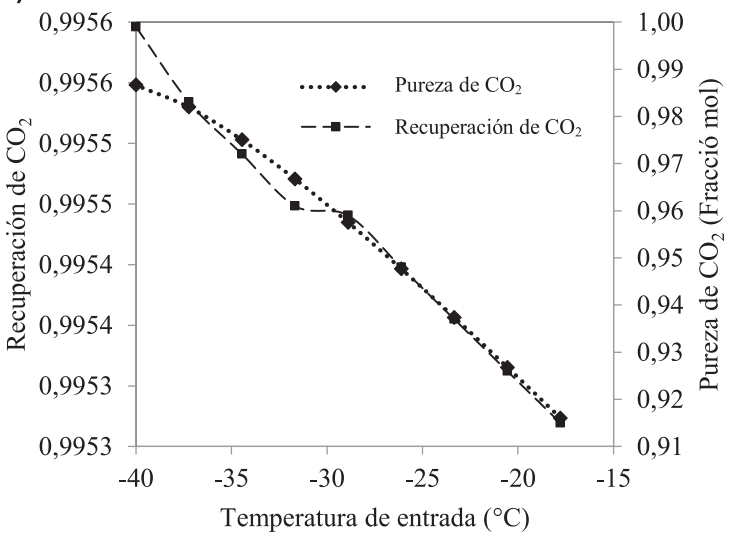

Figura 6. Efecto de (a) la presión de la corriente de gas de entrada y (b) la presión de la temperatura del condensador sobre la recuperación y la pureza de $\mathrm{CO}_{2}$ durante la simulación del proceso Ryan Holmes.

\subsection{Recuperación y pureza de $\mathrm{CO}_{2}$ de las tecnologías simuladas}

El objetivo de este estudio fue proponer un proceso de purificación de la corriente de $\mathrm{CO}_{2}$ desechada en los sistemas de endulzamiento de gas, que permita su posterior uso en la industria alimenticia, donde el $\mathrm{CO}_{2}$ debe tener una pureza superior al 99,95\%. La Tabla 2 muestra los resultados de máximas recuperación y pureza de $\mathrm{CO}_{2}$ obtenidos con cada tecnología, además de su comparación con otras tecnologías reportadas. El Proceso 1: Eliminación de $\mathrm{H}_{2} \mathrm{~S}$ por absorción con aminas arrojó valores de pureza y recuperación de $\mathrm{CO}_{2}$ considerablemente elevadas. El Proceso 2: Deshidratación con TEG, que incluyó un tamiz molécular, entregó una pureza de $\mathrm{CO}_{2}$ de $99,81 \%$, con una recuperación de $99,74 \%$. La adsorción con tamiz molécular se ha reportado con recuperaciones del 95\% [17], superadas en este estudio por su combinación con un sistema de absorción con TEG. Las mayores recuperación y pureza de $\mathrm{CO}_{2}$ se obtuvieron con el Proceso 3: Sistema criogénico Ryan Holmes (99,91 y 99,90\%, respectivamente). La recuperación del Proceso 3 se ha reportado como superior al $95 \%$ y produce $\mathrm{CO}_{2}$ líquido de alta pureza [17]. Sin embargo, ninguna de las tecnologías alcanzó los estándares requeridos por la industria alimenticia de manera aislada. Por ello, se diseñaron combinaciones para aumentar la pureza de la corriente de $\mathrm{CO}_{2}$. La combinación (1) integró los procesos 1 y 2 , finalizados con un proceso de compresión. Esta combinación arrojó una corriente de $\mathrm{CO}_{2}$ con una pureza de $99.83 \%$ y una recuperación de $99,85 \%$, debido a la combinación de la absorción y la adsorción. La capacidad del sistema de absorción depende de la saturación de gas de TEG; sin embargo, la combinación del sistema de deshidratación y el tamiz molécular es significativamente eficaz en la purificación. No obstante, la combinación aún no entrega una corriente lo suficientemente pura para la industria alimenticia. Esta corriente podría ser usada en el recobro mejorado del petróleo en campos de la misma planta de gas [2]. Finalmente, la combinación 2, que incluye los tres sistemas simulados, permite obtener una corriente de $\mathrm{CO}_{2}$ con la pureza requerida por la industria alimenticia en la fase líquida. Además, el $\mathrm{CO}_{2}$ producido en fase gaseosa se puede comercializar para usos de menor pureza. 
Tabla 2. Eficiencia y costos de los procesos simulados

\begin{tabular}{|c|c|c|c|c|c|}
\hline Proceso & $\begin{array}{l}\text { Pureza } \\
\text { de } \mathrm{CO}_{2} \\
(\mathrm{~mol} \%)\end{array}$ & $\begin{array}{l}\text { Recuperación de } \\
\mathrm{CO}_{2}(\%)\end{array}$ & $\begin{array}{l}\text { Flexibilidad de } \\
\text { Operación }\end{array}$ & $\begin{array}{l}\text { Costo total } \\
\text { (USD) }\end{array}$ & $\begin{array}{l}\text { Costo norma- } \\
\text { lizado (USD/ } \\
\left.\mathrm{tCO}_{2}\right)\end{array}$ \\
\hline $\begin{array}{l}\text { 1. Eliminación de } \mathrm{H}_{2} \mathrm{~S} \text { por } \\
\text { absorción con aminas }\end{array}$ & 98,72 & 94,02 & Moderada & $\$ 32,129$ & $\$ 4$ \\
\hline $\begin{array}{l}\text { 2. Deshidratación con } \\
\text { TEG }\end{array}$ & 99,81 & 99,74 & Moderada & $\$ 89,772$ & $\$ 12$ \\
\hline $\begin{array}{l}\text { 3. Sistema criogénico } \\
\text { Ryan Holmes }\end{array}$ & 99,90 & 99,91 & Moderada & $\$ 158,430$ & $\$ 21$ \\
\hline $\begin{array}{l}\text { Combinación 1: Procesos } \\
1 \text { y } 2 \text { + Compresión }\end{array}$ & 99,83 & 99,85 & Moderada & $\$ 286,322$ & $\$ 37$ \\
\hline $\begin{array}{l}\text { Combinación 2: Procesos } \\
1,2 \text { y } 3 \text { + Compression }\end{array}$ & 99,95 & 99,99 & Baja & $\$ 412,623$ & $\$ 53$ \\
\hline
\end{tabular}

\begin{tabular}{lccccc}
\hline \multicolumn{5}{c}{ Otros estudios } \\
\hline Absorción & -- & $90,0-98,0[18]$ & Moderada [19] & -- & $\$ 42-61[20]$ \\
\hline Absorción & -- & $80,0-95,0[21]$ & Moderada [21] & -- \\
\hline Criogénico Ryan Holmes & -- & $>95,0[17]$ & Baja [18] & -- & \\
\hline
\end{tabular}

La flexibilidad operacional de las tres tecnologías se analizó con base en las condiciones de operación de los procesos diseñados. La Tabla 2 muestra dos niveles de flexibilidad operacional: moderada y baja. Los procesos 1 y 2 presentan flexibilidad moderada debido a que el control de las condiciones de operación es relativamente sencillo por la experiencia de la industria $O \& G$ en estos sistemas. Mientras que, en sistemas criogénicos como el Ryan Holmes, se requerirá una mayor supervisión debido a la manipulación de fluidos supercríticos, lo que la cataloga con una flexibilidad operacional baja.

\subsection{Costos de inversión}

La Tabla 2 muestra los costos de inversión de capital (CAPEX) de las tecnologías y sus combinaciones. Los valores se presentan como costo total en dólares (USD) y normalizados por tonelada de $\mathrm{CO}_{2}$ tratada [5]. Se observa que el aumento de pocos puntos porcentuales en la pureza del $\mathrm{CO}_{2}$ requiere aumentos en las inversiones de capital de más de un orden de magnitud. La combinación 1, aunque no logra la pureza requerida para la industria alimenticia, puede producir un $\mathrm{CO}_{2}$ apto para su reinyección en los pozos de petróleo y otros usos para diferentes industrias a un costo competitivo [20], con un valor de 37 $\mathrm{USD} / \mathrm{tCO}_{2}$. Los procesos que involucraron el sistema criogénico mostraron altos costos debido a los procesos de compresión y secado de gas a bajas temperaturas, donde el $\mathrm{CO}_{2}$ obtenido está en fase líquida. La combinación 2 es $31 \%$ más costosa que la combinación 1, pero entrega el $\mathrm{CO}_{2}$ requerido.

De esta manera, la corriente de $\mathrm{CO}_{2}$ emitida en las plantas de endulzamiento de gas puede tratarse por una combinación de sistemas de absorción con MDEA para retirar el $\mathrm{H}_{2} \mathrm{~S}$, de deshidratación con TEG para retirar la humedad y de purificación final por un proceso criogénico Ryan Holmes. Esta combinación de procesos tendría unos costos de inversión cercanos a 53 USD/ $\mathrm{tCO}_{2}$, para un $\mathrm{CO}_{2}$ que puede comercializarse a valores superiores a $5000 \mathrm{USD} / \mathrm{tCO}_{2}$. La tecnología de Oxy-combustión de $\mathrm{CO}_{2}$ ha reportado costos de $52 \mathrm{USD} / \mathrm{tCO}_{2}$, similar al resultante de la combinación 2. De la misma manera, la combinación 1 arroja un valor de $37 \mathrm{USD} / \mathrm{tCO}_{2}$, menor comparada con las tecnologías mencionadas, con un $\mathrm{CO}_{2}$ de pureza apta para variadas aplicaciones [20].

\section{CONCLUSIONES}

En este trabajo se simularon tres procesos para la purificación del $\mathrm{CO}_{2}$ emitido en una planta de tratamiento de gas natural. El Proceso 1, consistente de la eliminación de $\mathrm{H}_{2} \mathrm{~S}$ por absorción con 
aminas, produjo una pureza y una recuperación de $\mathrm{CO}_{2}$ de 98,72 y $94,02 \%$, respectivamente. El Proceso 2: Deshidratación con TEG combinado con tamiz molécular, entregó una pureza de $\mathrm{CO}_{2}$ de $99,81 \%$ y una recuperación de $99,74 \%$. El Proceso 3: Sistema criogénico Ryan Holmes entregó una recuperación y una pureza de $\mathrm{CO}_{2}$ de 99,91 y $99,90 \%$, respectivamente. Debido a que ninguna tecnología por sí sola entregó el objetivo de pureza de $\mathrm{CO}_{2}$ de $99,95 \%$ mol para su uso en la industria alimenticia, se ensayaron diferentes combinaciones. La combinación de las tres tecnologías permite obtener un $\mathrm{CO}_{2}$ al $99.95 \% \mathrm{~mol}$ en la fase líquida del proceso. Los costos de las tecnologías oscilaron entre 4 y $53 \mathrm{USD} / \mathrm{tCO}_{2}$, relacionados directamente con la pureza obtenida.

De esta manera, la purificación del $\mathrm{CO}_{2}$ emitido en plantas de endulzamiento de gas natural por la combinación de sistemas de endulzamiento con MDEA, deshidratación con TEG y sistema criogénico Ryan Holmes, puede entregar corrientes de $\mathrm{CO}_{2}$ de alta pureza $(99,99 \%)$ que puede ser usado en la industria alimenticia, con un costo de inversión comparable a otras tecnologías de captura de $\mathrm{CO}_{2}\left(53 \mathrm{USD} / \mathrm{tCO}_{2}\right)$.

\section{AGRADECIMIENTOS}

Los autores agradecen a la Universidad de La Sabana por su apoyo en la financiación del proyecto ING-192-2016. N. Gámez agradece la empresa Stork (MASA) por facilitar la información para este proyecto y el apoyo a sus estudios de maestría.

\section{REFERENCIAS}

[1] Comisión de Regulación de Energía y Gas. Resolución No. 071. Reglamento Único de Transporte de Colombia. Sesión No. 324. Especificaciones de calidad del gas natural en el punto de entrada del sistema de transporte [Online], 2007. Disponible en: http://bilateralenergy.com/Reglamento $\% 20$ Unico $\% 20 \mathrm{de} \% 20$ Transporte $\% 20 \mathrm{de} \% 20$ Gas\%20Natural.pdf
[2] A. Ahmed, M. Gavin, "Industrial Design Gas and Optimization of $\mathrm{CO}_{2}$ Capture, Dehydration, and Compression Facilities", Gas Processors Association Convention, Bryan Research \& Engineering [Online], 2009. Disponible en: https://www.bre. com/PDF/Industrial-DesingGas-and-Optimization-of- $\mathrm{CO}_{2}$-Capture-Dehydration-and-Compression-Facilities.pdf

[3] Gas Processors Association, Engineering Data Book, 12a ed, Tulsa, Oklahoma, 2004.

[4] B. Moreno, E.A. Campanella, "Simulation of the Chemical Absorption Process with Amines Solutions for Biogas Purification", Inf. tecnol. vol. 24, pp 25-32, 2013. doi: 10.4067/S0718-07642013000100004.32.

[5] C. Echeverry, "Estimación de la emisión de gases de efecto invernadero en el municipio de Montería (Córdoba, Colombia)", Revista Ingenierías Universidad de Medellín vol. 5. pp. 85-09, julio 2006. Disponible en: http:// www.scielo.org.co/pdf/rium/v5n9/v5n9a08. pdf

[6] H.M. Ribon, N. Santos, O.P. Ortiz, "Métodos de deshidratación de gas natural", Revista Fuentes: El Reventón Energético vol. 8, pp. 55-64, julio 2010.

[7] E.N. Aguilera, M.E. Sánchez, J.M. Ortiz, "Adsorbentes para la deshidratación de gas húmedo dulce: avances y tendencias", Tecnología Química vol. 33, pp. 47-63, enero 2013. Disponible en: http://revistas.uo.edu. cu/index.php/tq/article/viewFile/844/808

[8] A.J. Finn, Contain Natural Resources [Online], Processing of Carbon Dioxide Rich Gas. GPA Conference. Manchester, 2014. Disponible en: http://www.costain.com/ media/13020/gpa-conference-sept-20141mb. pdf.

[9] M.J. Martínez, Deshidratación de gas natural [Online], 2011, pp. 141-152. Disponible en: https://www.academia.edu/31232586/ Deshidratacion_del_gas_natural_marcias_martinez.pdf?auto=download 
[10] L. Addington, C. Ness. An evaluation of General "Rules of thumb" in amine Sweetening Unit design Gas and Operation [Online], Gas Processors Association Convention, Bryan Research and Engineering. Disponible en: http://www.eighbooks.com/ lib.php?q=chemical-engineering-7th-edition-solutions-manual\#!

[11] R. Perry Robert, G. Don Green, Maloney J, Manuel del Ingeniero Químico. 7a Ed. McGraw-Hill Education, 2001, 11.4.

[12] American Association of Cost Engineers [Online], 2015. Disponible en: http://www. aacei.org/.

[13] N. Berghout, M. van den Broek, A. Faaij, "Techno-economic performance and challenges of applying $\mathrm{CO} 2$ capture in the industry", Int. J. Greenhouse Gas Control vol. 17, pp. 259-279, September 2013. doi: 10.1016/j.jiggc.2013.04.022.

[14] W.D. Seider, J.D. Seader, D.R. Lewin, Product \& Process Design Gas Principles: Synthesis, Analysis and Evaluation, 3rd ed., Wiley, New Jersey, 2009.

[15] World Bank, 2017. Disponible en: http:// www.worldbank.org/.
[16] M. Netušil \& P. Ditl, "Natural Gas Dehydration" in Natural Gas - Extraction to End Use, S.B. Gupta, Ed. InTech, Octubre, 2012, pp. 4-22.

[17] M. Mondal, H. Balsora, P. Varshney, "Progress and trends in $\mathrm{CO}_{2}$ capture/ separation technologies", Energy vol. 46, pp. 431-441, October 2012. doi: 10.1016/j. energy.2012.08.006.441.

[18] S. Mamun, V.Y. Dindore, H.F. Svendsen, "Kinetics of the reaction of carbon dioxide with aqueous solutions of 2-((2-aminoethyl) amino) ethanol", 「Ind. Eng. Chem. Res vol. 46, pp 385-394, December 2007. doi: $10.1021 / \mathrm{ie} 060383 \mathrm{v}$.

[19] A.A. Olajire, "CO2 capture and separation technologies for end-of-pipe applications A review", Energy vol. 35, pp. 2610-2628, June 2010. doi: 10.1016/j.energy.2010.02.030.

[20] M. Finkenrath, Cost and Performance of Carbon Dioxide Capture from Power Generation, IEA Energy Pap. (2011) 51. doi:10.1002/ceat.201100444.

[21] A. Meisen, X. Shuai, "Research and development issues in $\mathrm{CO} 2$ capture", Energy Convers. Manag. Vol. 38, pp. S37-S42, 1997. Doi: 10.1016/S0196-8904(96)00242-7. 